\title{
Choice functions and rejection sets
}

\author{
Enrique Miranda ${ }^{1}$, Arthur Van Camp ${ }^{2}$, and Gert de Cooman ${ }^{2}$
}

\begin{abstract}
We establish an equivalent representation of coherent choice functions in terms of a family of rejection sets, and investigate how each of the coherence axioms translates into this framework. In addition, we show that this family allows to simplify the verification of coherence in a number of particular cases.
\end{abstract}

\section{Introduction}

Coherent choice functions constitute an uncertainty model that is more general than sets of desirable gambles, while still preserving some of their nice properties, such as being able to deal effectively with sets of probability zero when conditioning. One of their drawbacks is the technical difficulty of verifying the coherence axioms. In this paper, we try to remedy this situation somewhat by providing an equivalent representation of choice functions in terms of those option sets that allow a subject to reject the zero gamble, which may be interpreted as those option sets that he should consider preferable to the status quo. As we shall see, this representation, in addition to capturing more intuitively the ideas underlying coherence, also helps to simplify the verification of coherence in a number of particular cases.

This paper is organized as follows: in Section 2, we recall the basic aspects of coherent choice functions that we shall need in the rest of the paper. Our representation in terms of rejection sets is established in Section 3, where we also discuss two additional properties that seem of interest for choice functions. In Section 4, we look in more detail at a number of particular cases: choice functions on binary spaces (that is, when the experiment on which the outcomes of the options depend on can only take two values) and those defined by means of

University of Oviedo, Dep. of Statistics and Operations Research mirandaenrique@uniovi.es · Ghent University, IDLab arthur.vancamp@ugent.be and gert. decooman@ugent. be 
coherent sets of desirable gambles. The paper concludes with some additional remarks in Section 5.

\section{Coherent choice functions}

Let $\Omega$ be a possibility space. A gamble on $\Omega$ is a bounded map $f: \Omega \rightarrow \mathbb{R}$. We denote by $\mathscr{L}$, the set of all gambles on $\Omega$. Gambles will also be called options. For any two gambles $f$ and $g$, we denote $f \leq g$ if $f(\omega) \leq g(\omega)$ for every $\omega$ in $\Omega$, and we collect all the gambles $f$ for which $f \leq 0$ in $\mathscr{L}_{\leq 0}$. We let $f<g$ if $f \leq g$ and $f \neq g$, and collect all the gambles $f$ for which $f<0$ in $\mathscr{L}_{<0}$, and the gambles $f$ for which $f>0$ in $\mathscr{L}_{>0}$.

Choice functions are defined on finite collections of gambles. We collect all those collections in the set $\mathscr{Q}$.

Definition 1. A choice function $C$ on a possibility space $\Omega$ is a map

$$
C: \mathscr{Q} \rightarrow \mathscr{Q} \cup\{\varnothing\}: A \mapsto C(A) \text { such that } C(A) \subseteq A \text {. }
$$

We collect all the choice functions on $\Omega$ in $\mathscr{C}(\Omega)$, often denoted as $\mathscr{C}$ when the possibility space is clear from the context.

The idea underlying this simple definition is that a choice function $C$ selects the set $C(A)$ of 'best' options in the option set A. Our definition resembles the one commonly used in the literature (Aizerman, 1985; Seidenfeld et al., 2010; Sen, 1977), except perhaps for an also not entirely unusual restriction to finite option sets (He, 2012; Schwartz, 1972; Sen, 1971).

Equivalently to a choice function $C$, we may consider its associated rejection function $R$, defined by $R(A):=A \backslash C(A)$ for all $A$ in $\mathscr{Q}$. It returns the options $R(A)$ that are rejected-not selected-by $C$.

We focus here on a special class of choice functions, which we call coherent.

Definition 2. We call a choice function $C$ on $\Omega$ coherent if for all $A, A_{1}$ and $A_{2}$ in $\mathscr{Q}$, all $f$ and $g$ in $\mathscr{L}$, and all $\lambda$ in $\mathbb{R}_{>0}:^{1}$

$\mathrm{C}_{1} . C(A) \neq \varnothing$;

$\mathrm{C}_{2}$. if $f<g$ then $\{g\}=C(\{f, g\})$;

$\mathrm{C}_{3}$. a. if $C\left(A_{2}\right) \subseteq A_{2} \backslash A_{1}$ and $A_{1} \subseteq A_{2} \subseteq A$ then $C(A) \subseteq A \backslash A_{1}$;

b. if $C\left(A_{2}\right) \subseteq A_{1}$ and $A \subseteq A_{2} \backslash A_{1}$ then $C\left(A_{2} \backslash A\right) \subseteq A_{1}$;

$\mathrm{C}_{4}$. a. if $A_{1} \subseteq C\left(A_{2}\right)$ then $\lambda A_{1} \subseteq C\left(\lambda A_{2}\right)$;

b. if $A_{1} \subseteq C\left(A_{2}\right)$ then $A_{1}+\{f\} \subseteq C\left(A_{2}+\{f\}\right)$;

These axioms are a subset of the ones introduced in Seidenfeld et al. (2010), duly translated from horse lotteries to gambles. We have omitted two of the coherence axioms from Seidenfeld et al. (2010): one is the Archimedean axiom, be-

\footnotetext{
${ }^{1}$ By $\mathbb{R}_{>0}$ we mean all the (strictly) positive real numbers.
} 
cause it is not fully compatible with the idea of deriving choice functions from coherent sets of desirable gambles (Van Camp et al., 2015), which is one of the goals in our approach. The other one, which we shall consider later on, is the so-called convexity axiom. Although this axiom leads to a number of useful properties, and in particular to a connection with lexicographic probability systems (Van Camp et al., 2016), we have refrained from including it in the list of coherence axioms because it not satisfied by some interesting choice functions.

Equivalent formulations of these axioms, better suited for our subsequent proofs, are the following:

$$
\begin{aligned}
& \left(\mathrm{C}_{3} \mathrm{a}\right) \Leftrightarrow\left(\forall A, A^{\prime} \in \mathscr{Q}, \forall f \in A\right)\left(\left(f \in R(A), A \subseteq A^{\prime}\right) \Rightarrow f \in R\left(A^{\prime}\right)\right), \\
& \left(\mathrm{C}_{3} \mathrm{~b}\right) \Leftrightarrow(\forall A \in \mathscr{Q}, \forall f \in A)(\{0, f\} \subseteq R(A) \Rightarrow 0 \in R(A \backslash\{f\})), \\
& \left(\mathrm{C}_{4} \mathrm{a}\right) \Leftrightarrow(\forall A \in \mathscr{Q}, \forall \lambda>0) R(\lambda A)=\lambda R(A), \\
& \left(\mathrm{C}_{4} \mathrm{~b}\right) \Leftrightarrow(\forall A \in \mathscr{Q}, \forall f \in \mathscr{L}) R(A+f)=R(A)+f .
\end{aligned}
$$

\section{A representation in terms of rejection sets}

Next we give an equivalent representation of choice functions in terms of rejection sets. For any $f \in \mathscr{L}$ and any natural number $i$, we define

$$
\mathbb{K}_{f}^{i}:=\{A: f \in R(A),|A|=i\} \text { and } \mathbb{K}_{f}:=\cup_{i \in \mathbb{N}} \mathbb{K}_{f}^{i} .
$$

We are going to characterize coherent choice functions in terms of these rejection sets. Our first result shows that we can restrict our attention to the case $f=0$ :

Proposition 1. Let $C$ be a choice function and consider the family of option sets $\left\{\mathbb{K}_{f}: f \in \Omega\right\}$ it induces by means of Eq. (1). Then

$$
\text { C satisfies Axiom } \mathrm{C}_{4} \mathrm{~b} \Leftrightarrow(\forall f \in \mathscr{L}) \mathbb{K}_{0}+f=\mathbb{K}_{f} \text {. }
$$

Proof. For necessity, consider an option set $A$ that includes 0 . Then the option set $A+f$ includes $f$, and since by $\mathrm{C}_{4} \mathrm{~b}$ it holds that $R(A+f)=R(A)+f$, we conclude that $A \in \mathbb{K}_{0}$ if and only if $A+f \in \mathbb{K}_{f}$.

Conversely, for sufficiency, consider an option set $A$ and a gamble $f$. Take any $g \in R(A)$, then $A \in \mathbb{K}_{g}$, whence by assumption $A-g \in \mathbb{K}_{0}$ and as a consequence $A-g+(f+g)=A+f \in \mathbb{K}_{f+g}$. Then indeed $g+f \in R(A+f)$, whence Axiom $\mathrm{C}_{4} \mathrm{~b}$ holds.

Taking this result into account, in what follows we shall restrict our attention to rejection sets $\mathbb{K}$ for which $\mathbb{K}_{0}+f=\mathbb{K}_{f}$ for every $f$ in $\mathscr{L}$. We can then simplify the notation above to

$$
K^{i}:=\mathbb{K}_{0}^{i}=\{A: 0 \in R(A),|A|=i\} \text { and } K:=\mathbb{K}_{0}=\{A: 0 \in R(A)\},
$$


respectively, and denote $\mathscr{Q}_{0}$ the family of option sets that include the zero gamble. Our next result provides a characterisation of the different coherent axioms in terms of these sets:

Proposition 2. Let $C$ be a choice function satisfying Axiom $\mathrm{C}_{4} \mathrm{~b}$, and consider the sets $K^{i}, K$ defined in Eq. (2).

1. C satisfies Axiom $\mathrm{C}_{1}$ if and only if $\left(\forall A \in \mathscr{Q}_{0}\right)(\exists f \in A) A-f \notin K$.

2. $C$ satisfies Axiom $\mathrm{C}_{2}$ if and only if $\left(\forall f \in \mathscr{L}_{>0}\right)\{f, 0\} \subseteq K^{2}$.

3. $C$ satisfies Axiom $\mathrm{C}_{3}$ a if and only if $\left(\forall A \in K, \forall A^{\prime} \in \mathscr{Q}_{0}\right)\left(A \subseteq A^{\prime} \Rightarrow A^{\prime} \in K\right)$.

4. $C$ satisfies Axiom $\mathrm{C}_{3} \mathrm{~b}$ if and only if $(\forall A \in K, \forall f \in A)(A-f \in K \Rightarrow A \backslash\{f\} \in K)$.

5. C satisfies Axiom $\mathrm{C}_{4}$ a if and only if $\left(\forall A \in \mathscr{Q}_{0}, \forall \lambda>0\right)(A \in K \Leftrightarrow \lambda A \in K)$.

Proof. 1. Taking Axiom $\mathrm{C}_{4}$ b into account, Axiom $\mathrm{C}_{1}$ holds if and only if $C(A) \neq \varnothing$ for every $A \in \mathscr{Q}_{0}$. This in turn is equivalent to $(\exists f \in A) f \in C(A)$, which by $\mathrm{C}_{4} \mathrm{~b}$ is equivalent to $0 \in C(A-f)$ or, in other words, to $A-f \notin K$.

2. Under Axiom $\mathrm{C}_{4}$ b, Axiom $\mathrm{C}_{2}$ is equivalent to $\left(\forall f \in \mathscr{L}_{>0}\right)\{f\}=C(\{0, f\})$, or, in other words, to $\left(\forall f \in \mathscr{L}_{>0}\right)\{f, 0\} \subseteq K^{2}$.

3. For necessity, consider any $A$ in $K$ and any $A^{\prime}$ in $\mathscr{Q}_{0}$ such that $A^{\prime} \supseteq A$. Because $A \in K, 0 \in R(A)$, whence, by Axiom $\mathrm{C}_{3} \mathrm{a}, 0 \in R\left(A^{\prime}\right)$. Then indeed $A^{\prime} \in K$.

Conversely, for sufficiency, consider any $A$ and $A^{\prime}$ in $\mathscr{Q}_{0}$ such that $A \subseteq A^{\prime}$, and any $f$ in $R(A)$. Then by Axiom $\mathrm{C}_{4} \mathrm{~b}, 0 \in R(A-f)$, so $A-f \in K$, whence also $A^{\prime}-f \in K$, because $A^{\prime}-f \supseteq A-f$. Then $0 \in R\left(A^{\prime}-f\right)$, and applying again $\mathrm{C}_{4} \mathrm{~b}$, indeed $f \in R\left(A^{\prime}\right)$.

4. For necessity, consider any $A$ in $K$ and $f$ in $A$ such that $A-f \in K$. Then $0 \in$ $R(A-f)$, whence $f \in R(A)$, by Axiom $\mathrm{C}_{4} \mathrm{~b}$. Applying Axiom $\mathrm{C}_{3} \mathrm{~b}$, we deduce that $0 \in R(A \backslash\{f\})$, whence indeed $A \backslash\{f\} \in K$.

Conversely, for sufficiency, consider any $A$ in $\mathscr{Q}$ and $f$ in $A$ such that $\{0, f\} \subseteq$ $R(A)$. Then $A \in K$ and by Axiom $\mathrm{C}_{4} \mathrm{~b}, f \in R(A)$ implies that $0 \in R(A-f)$, so $A-f \in K$. Then $A \backslash\{f\} \in K$, or, in other words, indeed $0 \in R(A \backslash\{f\})$.

5. It suffices to note that under Axiom $\mathrm{C}_{4} \mathrm{~b}$, Axiom $\mathrm{C}_{4}$ a is equivalent to $0 \in C(A) \Leftrightarrow$ $0 \in C(\lambda A)$ for every $\lambda>0$ and every $A \in \mathscr{Q}_{0}$.

An immediate consequence is:

Corollary 1. A choice function $C$ is coherent if and only if it satisfies Axiom $\mathrm{C}_{4} \mathrm{~b}$ and the rejection set $K$ it induces by Eq. (2) is increasing, scale invariant, includes $\{f, 0\}$ for every $f \in \mathscr{L}_{>0}$ and it satisfies the following two properties:

- $\left(\forall A \in \mathscr{Q}_{0}\right)(\exists f \in A) A-f \notin K$.

- $(\forall A \in K, \forall f \in A)(A-f \in K \Rightarrow A \backslash\{f\} \in K)$.

Next we consider a couple of additional consistency axioms that were deemed interesting by Van Camp et al. (2016). The first one is the convexity axiom, which is given by:

$\mathrm{C}_{5}$. if $A \subseteq A_{1} \subseteq \mathrm{CH}(A)$ then $C(A) \subseteq C\left(A_{1}\right)$, for all $A$ and $A_{1}$ in $\mathscr{Q}$,

where $C(A):=\left\{\sum_{i=1}^{n} \alpha_{i} f_{i}: n \in \mathbb{N}, f_{i} \in A, \alpha_{i} \geq 0, \sum_{i=1}^{n} \alpha_{i}=1\right\}$ is the convex hull of $A$.

In terms of rejection sets, it is characterized by the following proposition: 
Proposition 3. Let $C$ be a choice function satisfying Axiom $\mathrm{C}_{4} \mathrm{~b}$. Then $C$ satisfies Axiom $\mathrm{C}_{5}$ if and only if $\left(\forall A_{1} \in K, \forall A \in \mathscr{Q}_{0}\right)\left(A \subseteq A_{1} \subseteq \mathrm{CH}(A) \Rightarrow A \in K\right)$.

Proof. For necessity, application of Axiom $\mathrm{C}_{5}$ tells us that, whenever $A \subseteq A_{1} \subseteq$ $\mathrm{CH}(A)$ hold, $0 \in R\left(A_{1}\right)$ implies that $0 \in R(A)$, or, in other words, $A_{1} \in K$ implies that $A \in K$.

Conversely, for sufficiency, consider two option sets $A$ and $A_{1}$ such that $A \subseteq$ $A_{1} \subseteq \mathrm{CH}(A)$, and let us show that $C(A) \subseteq C\left(A_{1}\right)$. Assume ex absurdo that there is some $f \in A$ such that $f \in R\left(A_{1}\right)$ and $f \in C(A)$. Then since $A-f \subseteq A_{1}-f \subseteq$ $\mathrm{CH}(A-f)$, we can apply axiom $\mathrm{C}_{4} \mathrm{~b}$ and assume that, without loss of generality, $f=0$. But then we obtain that $A_{1} \in K$ while $A \notin K$, a contradiction.

A weaker property that is also useful is the so-called separate homogeneity, which means that for all $n$ in $\mathbb{N}$, all $f_{1}, f_{2}, \ldots, f_{n}$ in $\mathscr{L}$ and all $\mu_{1}, \mu_{2}, \ldots \mu_{n}$ in $\mathbb{R}_{>0}$ :

$$
0 \in C\left(\left\{0, f_{1}, f_{2}, \ldots, f_{n}\right\}\right) \Leftrightarrow 0 \in C\left(\left\{0, \mu_{1} f_{1}, \mu_{2} f_{2}, \ldots, \mu_{n} f_{n}\right\}\right) .
$$

This property follows from axioms $\mathrm{C}_{3} \mathrm{a}, \mathrm{C}_{4} \mathrm{a}, \mathrm{C}_{5}$ (Van Camp et al., 2016, Proposition 1). Moreover, and unlike $\mathrm{C}_{5}$ that is linked to lexicographic choice functions, separate homogeneity is compatible with maximality as a decision rule, and therefore better suited for connecting choice functions with desirability. Furthermore, separate homogeneity is strictly weaker: there are classes of interesting coherent choice functions that satisfy Eq. (3) but not Axiom $\mathrm{C}_{5}$. In terms of the rejection sets, it is trivial to prove that it can be expressed in the following manner:

Proposition 4. Let C be a choice function satisfying Axiom $\mathrm{C}_{4} \mathrm{~b}$. It satisfies separate homogeneity if and only iffor all $n$ in $\mathbb{N}$, all $f_{1}, f_{2}, \ldots, f_{n}$ in $\mathscr{L}$ and all $\mu_{1}, \mu_{2}, \ldots \mu_{n}$ in $\mathbb{R}_{>0},\left\{0, f_{1}, f_{2}, \ldots, f_{n}\right\} \in K \Leftrightarrow\left\{0, \mu_{1} f_{1}, \mu_{2} f_{2}, \ldots, \mu_{n} f_{n}\right\} \in K$.

\section{Particular cases}

In this section, we consider a number of particular cases of choice functions for which the representation in terms of rejection sets simplifies somewhat.

\subsection{Coherent choice functions defined via maximality}

We begin by considering choice functions defined via Walley's notion of maximality (Van Camp et al., 2015). A set of gambles $\mathscr{D}$ is called coherent when it is a convex cone that includes all non-negative gambles and does not include the zero gamble. We refer to (Walley, 1991; Miranda and Zaffalon, 2010; Quaeghebeur, 2014) for a study of the notion of desirability and its variants. In particular, any coherent set of desirable gambles can be used to define a coherent choice function, by means of the formula 


$$
C_{\mathscr{D}}(A):=\{f \in A:(\forall g \in A) g-f \notin \mathscr{D}\} .
$$

Unlike general choice functions, the ones defined in the manner above are uniquely determined by binary comparisons. Thus, it is not surprising that for them the representation in terms of rejection sets takes a simpler form:

Proposition 5. Let $\mathscr{D}$ be a coherent set of gambles and let $C_{\mathscr{D}}$ be the coherent choice function it induces by Eq. (4). Then $K=\left\{A \in \mathscr{Q}_{0}:\left(\exists A_{1} \in K^{2}\right) A_{1} \subseteq A\right\}$ and $K^{2}=$ $\{\{0, f\}: f \in \mathscr{D}\}$.

Proof. Consider an option set $A$ in $K$. By Eq. (4), $0 \in R_{\mathscr{D}}(A)$ if and only if $A \cap$ $\mathscr{D} \neq \varnothing$. If $|A|=2$, then $A=\{0, f\}$ for some $f$ in $\mathscr{D}$, and as a consequence $K^{2} \supseteq$ $\{\{0, f\}: f \in \mathscr{D}\}$. Conversely, consider any $A^{\prime} \in K^{2}$. Then $A^{\prime}=\{0, g\}$ for some $g$ in $\mathscr{L}$. But since $0 \in R_{\mathscr{D}}\left(A^{\prime}\right)$, we have $g \in \mathscr{D}$, so $K^{2} \subseteq\{\{0, f\}: f \in \mathscr{D}\}$, proving that indeed $K^{2}=\{\{0, f\}: f \in \mathscr{D}\}$. If, on the other hand, $|A| \geq 3$, then $A \supseteq\{0, f\}$ for some $f$ in $\mathscr{D}$. But then $0 \in R_{\mathscr{D}}(\{0, f\})$, so $A \supseteq A^{\prime}$ for some $A^{\prime} \in K^{2}$, and therefore indeed $K=\left\{A \in \mathscr{Q}_{0}:\left(\exists A_{1} \in K^{2}\right) A_{1} \subseteq A\right\}$.

\subsection{Coherent choice functions on binary spaces}

Next, we consider coherent choice functions defined on binary spaces. It turns out that, under separate homogeneity, they are determined by rejection sets of cardinality two or three:

Proposition 6. Let $C$ be a coherent choice function on $\Omega=\{a, b\}$. If $C$ satisfies Eq. (3), then

$$
K=\left\{A \in \mathscr{Q}_{0}:\left(\exists A_{1} \in K^{2} \cup K^{3}\right) A_{1} \subseteq A\right\}
$$

Proof. Let us prove that for every $A$ in $K$ there exists a $A_{1}$ in $K^{2} \cup K^{3}$ for which $A_{1} \subseteq A$.

Consider thus $A$ in $K$. By Axiom $\mathrm{C}_{2}$, we find that $A \cap \mathscr{L}_{<0} \subseteq R\left(A \cap \mathscr{L}_{\leq 0}\right)$, so Axiom $\mathrm{C}_{3}$ a implies that then $A \cap \mathscr{L}_{<0} \subseteq R(A)$. Since $A \in K$ and therefore also $0 \in$ $R(A)$, by Axiom $\mathrm{C}_{3} \mathrm{~b}$ we find that then $0 \in\left(A \cap \mathscr{L}_{<0}^{c}\right)$, so we can assume without loss of generality that $A \cap \mathscr{L}_{<0}=\varnothing$. There are two possibilities.

If $A \cap \mathscr{L}_{>0} \neq \varnothing$, then for any $f$ in $A \cap \mathscr{L}_{>0}$ it follows from Axiom $\mathrm{C}_{2}$ that $0 \in$ $R(\{0, f\})$, whence the set $\{0, f\} \subseteq A$ belongs to $K^{2}$. So we find indeed that $A_{1}:=$ $\{0, f\}$ in $K^{2}$ for which $A_{1} \subseteq A$.

If $A \cap \mathscr{L}_{>0}=\varnothing$, then we can denote $A=\left\{f_{1}, \ldots, f_{n}, g_{1}, \ldots, g_{m}\right\}$ for some $n \geq$ 0 and $m \geq 0$ but $\max \{m, n\} \geq 1$, where $f_{i}$ belongs to the second quadrant (i.e., $\left.f_{i}(a)<0<f_{i}(b)\right)$ for every $i$ in $\{1, \ldots, n\}$ and $g_{j}$ belongs to the fourth quadrant (i.e., $\left.g_{j}(a)>0>g_{j}(b)\right)$ for every $j$ in $\{1, \ldots, m\}$. Let $\lambda_{i}:=\frac{-1}{f_{i}(a)}$ and $\mu_{j}:=\frac{1}{g_{j}(a)}$ for every $i$ in $\{1, \ldots, n\}$ and $j$ in $\{1, \ldots, m\}$. Then, applying Eq. (3),

$$
0 \in R\left(\left\{0, \lambda_{1} f_{1}, \ldots, \lambda_{n} f_{n}, \mu_{1} g_{1}, \ldots, \mu_{m} g_{m}\right\}\right)
$$


Infer that $\lambda_{i} f_{i}(a)=-1$ for every $i$ in $\{1, \ldots, n\}$. Letting $i^{*}:=\arg \max \left\{\lambda_{i} f_{i}(b): i \in\right.$ $\{1, \ldots, n\}\}$, we infer that

$$
\lambda_{k} f_{k}(b)<\lambda_{i^{*}} f_{i^{*}}(b) \Rightarrow \lambda_{k} f_{k} \in R\left(\left\{\lambda_{k} f_{k}, \lambda_{i^{*}} f_{i^{*}}\right\}\right) \Rightarrow \lambda_{k} f_{k} \in R(A),
$$

where last implication follows from Axiom $C_{3}$ a. similarly, $\mu_{j} g_{j}(a)=1$ for every $j$ in $1, \ldots, m$, and letting $j^{*}:=\arg \max \left\{\mu_{j} g_{j}(b): j \in\{1, \ldots, m\}\right\}$, we infer that

$$
\mu_{j} g_{j}(b)<\mu_{j^{*}} g_{j^{*}}(b) \Rightarrow \mu_{j} g_{j} \in R\left(\left\{\mu_{j} g_{j}, \mu_{j^{*}} g_{j^{*}}\right\}\right) \Rightarrow \mu_{j} g_{j} \in R(A),
$$

where again last implication follows from Axiom $\mathrm{C}_{3} \mathrm{a}$. If we now apply $\mathrm{C}_{3} \mathrm{~b}$, we deduce that $0 \in R\left(\left\{0, \lambda_{i^{*}} f_{i^{*}}, \mu_{j^{*}} g_{j^{*}}\right\}\right)$, whence $0 \in R\left(\left\{0, f_{i^{*}}, g_{j^{*}}\right\}\right)$, applying Eq. (3). Thus, there is a subset of $A$ with cardinality three that also belongs to $K$.

A key property in the proof of Proposition 6 is that separate homogeneity, together with Axiom $\mathrm{C}_{2}$, allows to assume without loss of generality that an option set $A$ that includes the zero gamble has at most one gamble $f$ in the second quadrant (for which $f(a)<0<f(b)$ ) and one $g$ in the fourth quadrant (for which $g(a)>0>g(b))$. Let us show that this need not happen without separate homogeneity:

Example 1. Consider $\Omega=\{a, b\}$ and let $\mathscr{D}$ be the coherent set of gambles $\mathscr{D}:=$ $\{f \in \mathscr{L}: f(a)<0<f(b)$ and $f(a)+f(b)>0\} \cup \mathscr{L}_{>0}$. Let $C$ be the choice function determined by the rejection function

$$
0 \in R(A) \Leftrightarrow\left(A \cap \mathscr{D} \neq \varnothing \text { or }\left(\exists \lambda_{1}>\lambda_{2}>0\right)\left\{\left(-\lambda_{1}, \lambda_{1}\right),\left(-\lambda_{2}, \lambda_{2}\right)\right\} \subseteq A\right)
$$

for all $A$ in $\mathscr{Q}_{0}$. We extend the domain of $R$ to $\mathscr{Q}$ by letting $f \in R(A) \Leftrightarrow 0 \in R(A-f)$ for all $A$ in $\mathscr{Q}$ and $f$ in $A$. Remark already that $(-\lambda, \lambda)$ lies on the border of $\mathscr{D}$ for every $\lambda>0$ : indeed, for every $g$ in $\mathscr{D}$ we have that $(-\lambda, \lambda)+g \in \mathscr{D}$.

Let us show that $C$ is a coherent choice function. Taking into account the last part of the definition, we see that $\mathrm{C}_{4} \mathrm{~b}$ holds, and we can restrict our attention to option sets in $\mathscr{Q}_{0}$. We show that $C$ satisfies Axioms $\mathrm{C}_{2}, \mathrm{C}_{3} \mathrm{a}, \mathrm{C}_{3} \mathrm{~b}, \mathrm{C}_{4} \mathrm{a}$, and $\mathrm{C}_{1}$, in this order.

For Axiom $\mathrm{C}_{2}$, consider any $f$ in $\mathscr{L}_{>0}$. Then $f \in \mathscr{D}$, so indeed $0 \in R(\{0, f\})$.

For Axiom $\mathrm{C}_{3} \mathrm{a}$, consider any $A$ and $A^{\prime}$ in $\mathscr{Q}_{0}$ such that $A \subseteq A^{\prime}$, and any $f$ in $R(A)$. Using Axiom $\mathrm{C}_{4} \mathrm{~b}$, then $0 \in R(A-f)$, whence $(A-f) \cap \mathscr{D} \neq \varnothing$ or $\left\{\left(-\lambda_{1}, \lambda_{1}\right),\left(-\lambda_{2}, \lambda_{2}\right)\right\} \subseteq A-f$ for some $\lambda_{1}>\lambda_{2}>0$. But $A^{\prime}-f \supseteq A-f$, so also $\left(A^{\prime}-f\right) \cap \mathscr{D} \neq \varnothing$ or $\left\{\left(-\lambda_{1}, \lambda_{1}\right),\left(-\lambda_{2}, \lambda_{2}\right)\right\} \subseteq A^{\prime}-f$, and therefore $0 \in R\left(A^{\prime}-f\right)$, whence, again by Axiom $\mathrm{C}_{4} \mathrm{~b}$, indeed $0 \in R\left(A^{\prime}\right)$.

For Axiom $\mathrm{C}_{3}$ b, consider any $A$ in $\mathscr{Q}_{0}$ and any $f$ in $A$ such that $\{0, f\} \subseteq R(A)$. We need to prove that then $0 \in R(A \backslash\{f\})$. Since $f \in R(A)$, then

(i) $\mathscr{D} \cap(A-f) \neq \varnothing$, or (ii) $\left\{\left(-\lambda_{1}, \lambda_{1}\right),\left(-\lambda_{2}, \lambda_{2}\right)\right\} \subseteq A-f$ for some $\lambda_{1}>\lambda_{2}>0$.

Furthermore, since $0 \in R(A)$, then $\mathscr{D} \cap A \neq \varnothing$, or $\left\{\left(-\lambda_{1}, \lambda_{1}\right),\left(-\lambda_{2}, \lambda_{2}\right)\right\} \subseteq A$ for some $\lambda_{1}>\lambda_{2}>0$. If $f \notin \mathscr{D} \cap A$ and $f \notin\left\{\left(-\lambda_{1}, \lambda_{1}\right),\left(-\lambda_{2}, \lambda_{2}\right)\right\}$, then also $\mathscr{D} \cap A \backslash\{f\} \neq \varnothing$ or 
$\left\{\left(-\lambda_{1}, \lambda_{1}\right),\left(-\lambda_{2}, \lambda_{2}\right)\right\} \subseteq A \backslash\{f\}$, whence $0 \in R(A \backslash\{f\})$. So assume that (a) $f \in \mathscr{D}$ or (b) $f=(-\lambda, \lambda)$ for some $\lambda>0$.

If (a) $f \in \mathscr{D}$, then (i) or (ii) must be the case. If (i) occurs, then there is some gamble $g$ in $(\mathscr{D}+f) \cap A$, whence $g-f \in \mathscr{D}$ for some $g$ in $A$. But since $f \in \mathscr{D}$, also $g=f+g-f \in \mathscr{D}$, and therefore $0 \in R(\{0, g\})$, whence by Axiom $\mathrm{C}_{3}$ a indeed $0 \in R(A \backslash\{f\})$. If (ii) occurs, then there are $\lambda_{1}>\lambda_{2}>0$ such that $f+\left(-\lambda_{1}, \lambda_{1}\right), f+$ $\left(-\lambda_{2}, \lambda_{2}\right) \in A$, whence, since $f \in \mathscr{D}$, by construction also $f+\left(-\lambda_{1}, \lambda_{1}\right) \in \mathscr{D}$. Therefore $0 \in R\left(\left\{0, f+\left(-\lambda_{1}, \lambda_{1}\right)\right\}\right)$, whence by Axiom $\mathrm{C}_{3}$ a, also $0 \in R(A \backslash\{f\})$.

If (b) $f=(-\lambda, \lambda)$ for some $\lambda>0$, then, similarly, (i) or (ii) must be the case. If (i) occurs, then there is some $g$ in $A$ such that $g-f \in \mathscr{D}$. Therefore by construction also $g=f+g-f \in \mathscr{D}$, whence $0 \in R(\{0, g\})$, and then by Axiom $\mathrm{C}_{3}$ a, also $0 \in R(A \backslash$ $\{f\}$ ). If (ii) occurs, then there is some $\lambda_{1}>0$ and $\lambda_{2}>0$ for which $\left\{f+\left(-\lambda_{1}, \lambda_{1}\right), f+\right.$ $\left.\left(-\lambda_{2}, \lambda_{2}\right)\right\}=\left\{\left(-\lambda-\lambda_{1}, \lambda+\lambda_{1}\right), f+\left(-\lambda-\lambda_{2}, \lambda+\lambda_{2}\right)\right\} \subseteq A$. Letting $\lambda_{1}^{\prime}:=\lambda+\lambda_{1}$ and $\lambda_{2}^{\prime}:=\lambda+\lambda_{2}$, we find that $\left\{\left(-\lambda_{1}^{\prime}, \lambda_{1}^{\prime}\right), f+\left(-\lambda_{2}^{\prime}, \lambda_{2}^{\prime}\right)\right\} \subseteq A \backslash\{f\}$, whence $0 \in R(A \backslash\{f\})$.

Axiom $\mathrm{C}_{4}$ a follows from Equation (5), taking into account that $\mathscr{D}$ is a cone.

Finally, for Axiom $\mathrm{C}_{1}$, assume ex absurdo that $C(A)=\varnothing$ for some $A$ in $\mathscr{Q}_{0}$. Then $A=R(A)$ whence, by Axiom $\mathrm{C}_{3} \mathrm{~b}, 0 \in R(\{0\})$. But $0 \notin \mathscr{D}$ and $(-\lambda, \lambda) \notin\{0\}$ for every $\lambda>0$, so $0 \notin R(\{0\})$, a contradiction.

On the other hand, it follows by Equation (5) that, given the option set $A=$ $\{0,(-1,1),(-2,2)\}$, we obtain $C(A)=\{(-1,1),(-2,2)\}$. However, the same equation implies that $0 \in C(\{0,(-1,1)\})$. This shows that $C$ does not satisfy separate homogeneity, and also that we cannot reduce the intersection with the second quadrant to only one gamble.

On the other hand, Proposition 6 also depends crucially on the assumption that $|\Omega|=2$, as our next example shows:

Example 2. Consider a ternary space $\Omega$, some $n$ in $\mathbb{N}$, and let $f_{k}$ be the gamble given by $f_{k}:=\left(-1, \frac{k}{n},-\frac{k^{2}}{n^{2}}\right)$, for all $k$ in $\{1, \ldots, n\}$. Let us show that for each $k$ we can find a probability measure whose expectation operator $P_{k}$ (called linear prevision in Walley's terminology) satisfies $P_{k}\left(f_{k}\right)>0>P_{k}\left(f_{j}\right)$ for every $j$ in $\{1, \ldots, n\} \backslash\{k\}$.

To find such expectation operators, let $P$ be the expectation operator associated with the mass function $\left(0, \frac{2 k}{n+2 k}, \frac{n}{n+2 k}\right)$. Then $P\left(f_{k}-f_{j}\right)=\frac{k-j}{n(n+2 k)}(2 k-(k+j))$, whence $P\left(f_{k}-f_{j}\right)>0$ if $k \neq j$. Moreover, $P\left(f_{k}\right)=\frac{k^{2}}{n(n+2 k)}>0$.

If we now consider any $\lambda \in(0,1)$ and define $P_{k}$ as the expectation operator associated with the mass function $\left(\lambda,(1-\lambda) \frac{2 k}{n+2 k},(1-\lambda) \frac{n}{n+2 k}\right)$, we obtain $P\left(f_{k}-\right.$ $\left.f_{j}\right)=(1-\lambda) P\left(f_{k}-f_{j}\right)>0$ whenever $k \neq j$. Moreover,

$$
P_{k}\left(f_{k}\right)=-\lambda+(1-\lambda) P\left(f_{k}\right)>0 \Leftrightarrow \lambda<\frac{P\left(f_{k}\right)}{1+P\left(f_{k}\right)},
$$

and similarly

$$
P_{k}\left(f_{j}\right)=-\lambda+(1-\lambda) P\left(f_{j}\right)<0 \Leftrightarrow \lambda>\frac{P\left(f_{j}\right)}{1+P\left(f_{j}\right)} .
$$


Since, for every $j$ in $\{1, \ldots, n\} \backslash\{k\}, \frac{P\left(f_{j}\right)}{1+P\left(f_{j}\right)}<\frac{P\left(f_{k}\right)}{1+P\left(f_{k}\right)}$ because $P\left(f_{j}\right)<P\left(f_{k}\right)$, we let

$$
\lambda \in\left(\max _{j \in\{1, \ldots, n\} \backslash\{k\}} \frac{P\left(f_{j}\right)}{1+P\left(f_{j}\right)}, \frac{P\left(f_{k}\right)}{1+P\left(f_{k}\right)}\right),
$$

and for this $\lambda$ we obtain $P_{k}\left(f_{k}\right)>0>P_{k}\left(f_{j}\right)$ for every $j \neq k$.

Now, let $\mathscr{D}_{k}$ be the coherent set of gambles given by $\mathscr{D}_{k}:=\left\{f \in \mathscr{L}: P_{k}(f)>0\right\}$, and let $C_{\mathscr{D}_{k}}$ be the coherent choice function it induces by Eq. (4). Then the choice function $C$ given by $C(A):=\bigcup_{k=1}^{n} C_{\mathscr{D}_{k}}(A)$ is also coherent (Van Camp et al., 2015, Proposition 3), and it can be checked to satisfy separate homogeneity because all $C_{\mathscr{D}_{k}}$ do. If we now consider the option set $A=\left\{0, f_{1}, \ldots, f_{n}\right\}$, we get that $C_{\mathscr{D}_{k}}(A)=$ $\left\{f_{k}\right\}$ for every $k$, since $P_{k}\left(f_{k}\right)>0>P_{k}\left(f_{j}\right)$ implies that $f_{k}, f_{k}-f_{j} \in \mathscr{D}_{k}$ for every $j$. As a consequence, we obtain $C(A)=\left\{f_{1}, \ldots, f_{n}\right\}$, whence $A \in K$. However, for every $k$ it holds that $C_{\mathscr{D}_{k}}\left(A \backslash\left\{f_{k}\right\}\right)=\{0\}$, using again that $P_{k}\left(f_{j}\right)<0$ for every $j \neq k$, and therefore $C\left(A \backslash\left\{f_{k}\right\}\right)=A \backslash\left\{f_{k}\right\}$. Thus, $A$ has no proper subset that also belongs to the rejection class $K$.

\section{Conclusions}

It is a consequence of coherence that a choice function is uniquely determined by those option sets that allow us to reject the zero gamble, i.e., those that are considered preferable to the status quo. In this paper, we have investigated the structure of these sets and shown that the coherence axioms can be expressed more intuitively in terms of these sets. In addition, we have shown that all the necessary information is given by option sets of cardinality two when the choice function is defined via maximality, and with cardinality two or three in most (but not all) cases of interest when the possibility space is binary. Moreover, we have shown that this last result does not extend to larger possibility spaces; thus, determining an analogous representation for arbitrary spaces would be the main open problem for the future.

Acknowledgements This paper was written during a stay from Arthur van Camp at the University of Oviedo, funded by Banco Santander via Campus de Excelencia Internacional. We would like to acknowledge this funding, as well as that of project TIN2014-59543-P. Gert de Cooman's research was partly funded through project number 3G012512 of the Research Foundation Flanders (FWO).

\section{References}

Aizerman M (1985) New problems in the general choice theory. Soc Cho Welf, 2

(4):235-282 
He J (2012) A generalized unification theorem for choice theoretic foundations: Avoiding the necessity of pairs and triplets. Economics Discussion Paper 2012-23, Kiel Institute for the World Economy. URL http://www.economicsejournal.org/economics/discussionpapers/2012-23.

Miranda E, Zaffalon M (2010) Notes on desirability and coherent lower previsions. Ann Math Art Intel 60(3-4):251-309

Quaeghebeur E (2014) Desirability. In Augustin T, Coolen F, de Cooman G, Troffaes $M$ (eds) Introduction to Imprecise Probabilities, chapter 1, pages 1-27. John Wiley \& Sons, Chichester

Schwartz T (1972) Rationality and the myth of the maximum. Noûs, 6(2):97-117

Seidenfeld T, Schervish M, Kadane J (2010) Coherent choice functions under uncertainty Synt 172(1):157-176

Sen A (1971) Choice functions and revealed preference. Rev Econ Stud 38(3): 307-317

Sen A (1977) Social choice theory: A re-examination. Econ 45:53-89

Van Camp A, de Cooman G, Miranda E, Quaeghebeur E (2015) Modelling indifference with choice functions. In Augustin T, Doria S, Miranda E, Quaeghebeur E (eds.), ISIPTA '15: Proceedings of the Ninth International Symposium on Imprecise Probability: Theories and Applications, 305-314. Aracne, Pescara

Van Camp A, Miranda E, de Cooman G (2016) Lexicographic choice functions without Archimedeanicity. In Ferraro M, Giordani P, Vantaggi B, Gagolewski M, Gil M A, Grzegorzewski P, Hryniewicz O (eds) Soft methods for data science, 479-486. Springer

Walley P (1991) Statistical Reasoning with Imprecise Probabilities. Chapman and Hall, London

\section{On Pedro Gil}

I was lucky to meet Pedro Gil in many different roles: first as a teacher, then as head of department, later as a supervisor, and finally, and all throughout, as a friend. He helped me in my first research work, on robust statistics for my final project during my BsC; and we taught together in a course on Mathematics for everyday life until a few weeks prior to his death.

He was brilliant as a professor, generous as a researcher, and charismatic as a leader. Few bring together unanimity in the manner he did; and still, or perhaps because of it, he was always unassuming: he would treat you the in the same manner irrespective of your position, and would make you feel that, whatever your problems, someone cared and would try to help. Because above all his many qualities, he had one that few possess: he was a good man.

He has not left a void because he is still very much present. 